\title{
Searching for Selective Scaffolds against Plasmodium falciparum Glucose-6-Phosphate Dehydrogenase 6-Phosphogluconolactonase ${ }^{+}$
}

\author{
Antonio Viayna 1,*, David Vílchez ${ }^{1}$, Javier Vázquez ${ }^{1,2}$, Susana Pérez-Benavente ${ }^{3}$, \\ José Manuel Bautista ${ }^{3,4}$ and Francisco Javier Luque ${ }^{1}$ \\ 1 Department of Nutrition, Food Science and Gastronomy, Faculty of Pharmacy and Food Sciences, Institute \\ of Biomedicine (IBUB) and Institute of Theoretical and Computational Chemistry (ITQCUB), University of \\ Barcelona, Av. Prat de la Riba 171, 08921 Santa Coloma de Gramenet, Spain \\ 2 Pharmacelera, Plaça Pau Vila, 1, Sector C 2a, Edifici Palau de Mar, 08039 Barcelona, Spain \\ 3 Department of Biochemistry and Molecular Biology, Universidad Complutense de Madrid, \\ Facultad de Veterinaria, Ciudad Universitaria, 28040 Madrid, Spain \\ 4 Research Institute Hospital 12 de Octubre, Avda. de Cordoba s/n, 28041 Madrid, Spain \\ * Correspondence: toniviayna@ub.edu \\ + Presented at the 2nd Molecules Medicinal Chemistry Symposium (MMCS): Facing Novel Challenges in \\ Drug Discovery, Barcelona, Spain, 15-17 May 2019.
}

Published: 7 August 2019

Keywords: Malaria; Plasmodium falciparum; Glucose-6-Phosphate Dehydrogenase 6Phosphogluconolactonase

Malaria is a parasitic disease caused by Plasmodium spp., being one of the major causes of death worldwide with two-hundred million new infections and hundreds of thousands of deaths in 2015. Despite the important advances in its prevention and treatment, its resistance to current drug therapies is still a serious risk in its eradication.

There is urgency in finding novel targets and drugs operating by novel mechanisms, avoiding cross-resistance to classical antimalarials. In this context, the bifunctional enzyme Glucose-6phosphate dehydrogenase 6-phosphogluconolactonase appears to be a promising therapeutic target due to its crucial role in regulating the PPP pathway (pentose phosphate pathway), which is the major source of redox potential in Plasmodium falciparum.

In the last few years, our group detected a specific mutation between the human and the Plasmodium falciparum form in the binding site of Glucose-6-phosphate (G6P), the endogenous ligand of Glucose-6-phosphate dehydrogenase (G6PD). This mutation involves the substitution of an Arginine (human) by an Aspartate (parasite), which allowed us to create a validated in-house homology model of PfG6PD.

Based on this result, the group has focused their efforts, through different molecular modelling techniques, in the discovery of selective scaffolds against PfG6PD. Current efforts address the development of a complete structural model of the bifunctional enzyme, which may offer novel opportunities to develop molecules capable of inhibiting this relevant enzyme.

(C) 2019 by the authors. Submitted for possible open access publication under the terms and conditions of the Creative Commons Attribution (CC BY) license (http://creativecommons.org/licenses/by/4.0/). 